fight AIDS, Tuberculosis and Malaria. However, additional implementation tools are required to support strengthening of STI-specific services for key populations.

Disclosure of interest statement Nothing to declare.

\section{P12.09 HIV CASCADE OF CARE: IMPROVEMENTS IN LINKAGE TO CARE AT THE STI CLINIC OF THE PUBLIC HEALTH SERVICE ROTTERDAM-RIJNMOND, THE NETHERLANDS}

\begin{abstract}
${ }^{1,2} \mathrm{HM} \mathrm{Götz}^{*},{ }^{1} \mathrm{MWH}$ Mattijsen, ${ }^{3} \mathrm{LM}$ van Zonneveld, ${ }^{4} \mathrm{JV}$ Smit, ${ }^{5} \mathrm{AA}$ van der Eijk, ${ }^{1,2} \mathrm{JH}$ Richardus. ${ }^{1}$ Public Health Service Rotterdam-Rijnmond, Rotterdam, The Netherlands; ${ }^{2}$ Department of Public Health, Erasmus MC, University Medical Center Rotterdam, The Netherlands; ${ }^{3}$ Department of Infectious Diseases, Erasmus MC, University Medical Center Rotterdam, The Netherlands; ${ }^{4}$ Department of Infectious Diseases, Maasstad Hospital Rotterdam, The Netherlands; ${ }^{5}$ Department of Viroscience, Erasmus MC, University Medical Center Rotterdam, The Netherlands
\end{abstract}

\subsection{6/sextrans-2015-052270.489}

Background The hiv cascade of care includes steps from testing to treatment. Once diagnosed, there are several steps determining the time between testing and linkage to care: Algorithm of hiv tests, communication of test results, way of referral to hiv treatment centre, and confirmation of being in care. In the course of 2010-2015 processes have been changed in the STI clinic to improve linkage to care.

Objectives To evaluate the efficiency of referral to the HIV treatment centres in Rotterdam we investigated the time between date of hiv test, date of referral to and first consultation at the hospital.

Methods We followed newly diagnosed patients from January 2010-March 2015 until linkage to care and collected data on time of HIV testing, discussing diagnosis, referral to and first consultation in care. Median time was calculated between testing, referral and first consultation in care, and regression analysis performed.

Results We identified 227 newly diagnosed patients, of which six refused referral, nine were referred to hospitals outside Rotterdam, and 212 were referred to an hiv-treatment centre in Rotterdam. 41 patients (19\%) were lost to follow up, 37 (43\%) between 2010 and 2012 vs. 4 (5\%) between 2013 and 2015. Of the 171 persons in care, the mean time between hiv test and arrival in hospital was 32 days, and decreased significantly $(\mathrm{p}=$ 0.004); median time was 39 days in 2010 and 14 days in 2015. The mean time between testing and referral was 18 days and decreased significantly ( $<<0.001$ ); (range median $22-9$ days). There was no decrease in time between referral and arrival in hospital.

Conclusion Time to entry into care can be improved in cooperation between STI clinic, laboratory and HIV treatment centre. Active follow-up for those referred is needed to facilitate interventions for entry into care.

Disclosure of interest statement No grants were received in the development of this study.

\section{P12.10 CHALLENGES IN IMPLEMENTING A PARTNER NOTIFICATION WEBTOOL IN GP PRACTICES IN THE NETHERLANDS: PRELIMINARY RESULTS OF A PILOT STUDY}

1,2 HM Götz* ${ }^{*}{ }^{1} J C M$ Watzeels, ${ }^{3,4}$ van Bergen Jeam, ${ }^{1,2}$ Voeten Hacm. ${ }^{1}$ Department Infectious Disease Control, Public Health Service Rotterdam-Rijnmond, Rotterdam, The Netherlands; ${ }^{2}$ Department of Public Health, Erasmus MC, University Medical Center Rotterdam, The Netherlands; ${ }^{3}$ Department of General Practice, Academic Medical Centre, University of Amsterdam, The Netherlands; ${ }^{4}$ Soa Aids Nederland, Amsterdam, The Netherlands

\section{$10.1136 /$ sextrans-2015-052270.490}

Introduction After evaluation of an internet-based partner notification (PN) system for verified diagnoses of STI/HIV, that uses an index-chosen method per partner (email, text messaging; (non-)anonymous) in STI clinics, ${ }^{1}$ we started a pilot project with General practitioners (GP) with www.partnerwaarschuwing.nl. The webtool includes training tools for GPs and practice assistants performing STI consultations and video instructions for patients.

Our aim was to evaluate the use of the webtool by GP practices as compared to STI clinics.

Methods We evaluated use of the web tool by GPs in the pilot project from April 2014 - March 2015. Numbers of created codes per professional, and numbers/method of sent notifications as well as login's by notified partners were extracted from the notification database.

Results 18/78 (23\%) professionals in GP practice who had applied for the pilot project during the year actually used the webtool. Also the Rotterdam and Amsterdam Public Health STI clinics used the webtool. 137 index-clients received a code by 18 professionals in GP practice (11 GPs, 7 practice assistants); mean number of codes provided $7.6 / 0.75$ year. $80 \%$ of the codes were provided by practice assistants. Of the GP's patients who received a code, $15 \%$ (21) notified contacts and sent 48 notifications (mean 2.3 ), $73 \%$ by text messaging and $27 \%$ by email. For 1010 STI clinic patients these numbers were 30\% (300), 961 notifications (mean 3.2), 95\% by text messaging. Of all notifications $86 \%$ was sent anonymously. Sixty-four percent of the partners notified checked their notification at the website (GP patient: 52\% [25/48] versus STI clinic 65\% [622/961].

Conclusion PN in GP practice is challenging. Improvement of $\mathrm{PN}$ in GP practice can be assisted by this webtool, especially in STI consultations by practice assistants. Further analysis of constraints for PN are ongoing to develop a multifaceted implementation strategy and strengthen $\mathrm{PN}$ in general practice.

Disclosure of interest statement This study was financed by ZonMW (project number 50-51515-98-243) and Stichting Coolsingel. No pharmaceutical grants were received in the development of this study.

\section{REFERENCE}

1 Gotz HM, van Rooijen MS, Vriens $P$, et al. Initial evaluation of use of an online partner notification tool for STI, called 'suggest a test': a cross sectional pilot study. Sex Transm Infect. 2014;90(3):195-200 